\title{
THEORETICAL INVESTIGATION OF THE MOLECULAR STRUCTURE AND MOLECULAR DOCKING OF ETORICOXIB
}

\author{
KANDASAMY SADASIVAM ${ }^{1}$, GUILLERMO SALGADO MORAN ${ }^{2}$, LUIS HUMBERTO MENDOZA-HUIZAR ${ }^{3 *}$, \\ WILSON CARDONA VILLADA ${ }^{4}$, LORENA GERLI CANDIA ${ }^{5}$, LORENA MARIBEL MENESES-OLMEDO ${ }^{6}$ \\ AND SEBASTIÁN CUESTA HOYOS ${ }^{6}$
}

\author{
${ }^{1}$ Department of Physics, Bannari Amman Institute of Technology, Sathyamangalam, Tamil Nadu, India 638401. \\ ${ }^{2}$ Facultad de Ciencias Físicas y Matemáticas, Universidad de Chile, Santiago. Chile. \\ ${ }^{3}$ Universidad Autónoma del Estado de Hidalgo. Academic Area of Chemistry. Carretera Pachuca-Tulancingo Km. 4.5 Mineral de la Reforma, Hgo., México. \\ ${ }^{4}$ Departamento de Ciencias Químicas, Facultad de Ciencias Exactas, Universidad Andrés Bello, Talcahuano, Chile. \\ ${ }^{5}$ Departamento de Química Ambiental, Facultad de Ciencias, Universidad Católica de la Santísima Concepción, Concepción, Chile. \\ ${ }^{6}$ Laboratorio de Química Computacional, Facultad de Ciencias Exactas y Naturales, Pontificia Universidad Católica del Ecuador, Quito, Ecuador.
}

\begin{abstract}
In this work, a computational chemical study of Etoricoxib was carried out at the X/6311G(d,p) (where X=B3LYP, M06 and $\omega$ B97XD) level of theory, at the gas, aqueous and ethanol phases. Through the chemical reactivity descriptors derived from the DFT, it was possible to find that Etoricoxib structure exhibits a major chemical activity in water and ethanol phases in comparison to the gas phase, which suggests this drug would be more active in biological solvents like in blood, tissues and places where the ciclooxigenasa 2 (COX)-2 is found. In addition, a molecular docking analysis was conducted to study the interaction of Etoricoxib with the COX-2 active site. The results suggest that Etoricoxib interacts with 19 amino acid residues inside the COX-2 active site.
\end{abstract}

Keywords: Etoricoxib; activity; DFT; COX-2; docking.

\section{INTRODUCTION}

Etoricoxib, [5-chloro-2-(6-methylpyridin-3-yl)-3-(4-methylsulfonylphenyl) pyridine], is a nonsteroidal anti-inflammatory drug (NSAID) prescribed as analgesic and anti-inflammatory. ${ }^{1-4}$ As a selective Cyclooxygenase-2 (COX-2) inhibitor, this lipophilic drug is taken orally to treat inflammatory conditions such as rheumatoid arthritis, gouty arthritis, osteoarthritis, musculoskeletal and menstrual pain. The recommended dosage prescribed by medical doctors goes from 60 to $120 \mathrm{mg} /$ day coming in 3 presentations $(60,90,120 \mathrm{mg} / \text { tablet) })^{1,2,5,6}$ Etoricoxib (ETX) is considered a medium size drug with a molecular weight of $358.84 \mathrm{~g} / \mathrm{mol}$. It is synthesized from vinamidinium salts. ${ }^{1,3}$ Its physical properties and stability can be defined by five ETX polymorphs reported in the literature. ${ }^{1,7,8}$ Also, this drug has been studied, in the materials science, for preparing densified glasses and ultra-stable materials. ${ }^{49}$ ETX has a good conformational flexibility, low and highly $\mathrm{pH}$-dependent water solubility $\left(3.28 \times 10^{-3} \mathrm{~g} \mathrm{~L}^{-1}\right)$, a $\log \mathrm{P}$ of 3.14 and a pKa of 4.6 behaving like a weak base. ${ }^{2}$ In this sense, there are several reports in the literature, which try to synthesize it in an amorphous form to overcome its water solubility issues. ${ }^{6}$ In vitro and in vivo studies have shown a rapid absorption and a good bioavailability of ETX when taken orally. ${ }^{2,10}$ Here, it is important to mention that ETX is part of the coxib family, which includes other related compounds such as celecoxib, valdecoxib and rofercoxib. This type of drugs has arisen a concern in regards to their safety, because a high number of cardiovascular events have been associated with the intake of these drugs. ${ }^{6,11}$ However, ETX has shown that is able to inhibit COX-2 but also neurodegeneration, neuroinflammation and memory impairment. Also, is able to reduce neuroinflammation in the hippocampus produced by colchicine. ${ }^{12}$ Furthermore, studies have demonstrated that this molecule exhibits antioxidant and anti-inflammatory properties, suggesting its benefits in temporary middle cerebral artery occlusion-related ischemia, testicular and ovarian ischemia reperfusion damage. ${ }^{5,13,14}$ Here, it is interesting to mention that the antioxidant activity of a compound is measured by their ability/capacity of transferring a hydrogen atom or an electron to reactive species..$^{15}$ Thus, electronic parameters such as: electronegativity, electrophilic index, hardness, softness, ionization potential, bond dissociation enthalpy and electron affinity may be used to evaluate this capacity. In this sense, the Density Functional Theory (DFT) has been used to analyze the relation between the structure of some compounds, identified as antioxidants, and their scavenging activity through the use of these electronic parameters. ${ }^{16-20}$

To the best of our knowledge, an analysis of the chemical reactivity of ETX at the molecular level has not been reported, yet. Therefore, in order to gain a deeper insight into this system, we analyze the chemical behavior of ETX through electronic parameters derived from the DFT. Also, a docking study is performed to analyze the binding conformation of ETX in the active site of COX-2.

\section{COMPUTATIONAL DETAILS}

\section{Molecular Descriptors}

All the calculations were carried out at the $\mathrm{X} / 6-311 \mathrm{G}(\mathrm{d}, \mathrm{p}$ ) (where

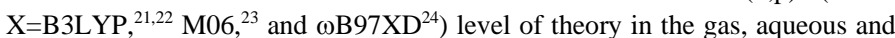
ethanolic phases, employing the Gaussian 09 package. ${ }^{25}$ The molecular descriptors such as: ionization potential (IP), electron affinity (EA), chemical hardness $(\eta)$, softness $(S)$ and electrophilicity index $(\omega)$ were evaluated because they are able to predict the general chemical behavior of a molecular system. ${ }^{26}$ Into the DFT scheme, IP was calculated as the energy difference between the compound derived from electron-transfer (cation) and the respective neutral compound. ${ }^{27}$

$\mathrm{IP}=E_{\text {cation }}-E_{\mathrm{n}}$

and EA as the energy difference between the neutral and anionic molecular system, ${ }^{27}$

$\mathrm{EA}=E_{\mathrm{n}}-E_{\text {anion }}$

Then, the parameters $\mu, \eta, S$ and $\omega$ were evaluated by using the following equations, ${ }^{28-31}$.

$$
\begin{aligned}
& \mu=-(\mathrm{IP}+\mathrm{EA}) / 2 \\
& \eta=(\mathrm{IP}-\mathrm{EA}) / 2 \\
& \mathrm{~S}=1 /(2 \eta) \\
& \omega=\mu^{2} / 2 \eta
\end{aligned}
$$

\section{Molecular Docking}

For the docking study, the $2.70 \AA$ resolution X-ray crystallographic structure of Rofecoxib bound to Homo sapiens COX-2 was used (PDB: 5KIR). The structure was prepared for the docking calculations using Pymol, ${ }^{32}$ Chimera 1.11.2, ${ }^{33}$ and Autodock Tools. ${ }^{34}$ In the preparation, as COX-2 is presented as a dimer, Chain B was eliminated. Structures not belonging to the enzyme such as phosphate ions, glycerol, alpha D-mannose, ammonium ions, and $\mathrm{N}$-acetyl-Dglucosamine were also eliminated. Two enzymatic models were built, one leaving all the water molecules and the other eliminating them. Finally, Rofecoxib structure occupying the active site of Chain A was removed. 
The ligand ETX used for the calculation was obtained from the DrugBank database (DB01628) ${ }^{35}$ Rofecoxib (RFX) structure was extracted from the crystal structure, validated by comparing it with the molecular structure found in the DrugBank database, and docked to COX-2 structure.

Molecular docking was performed using Autodock VINA. ${ }^{36}$ In the calculations, spacing was set to $1 \AA$, exhaustiveness to 8 , and the grid box was reduced to the active site. LigPlot,$+{ }^{37}$ Pymol,${ }^{32}$ and Autodock Tools, ${ }^{34}$ were used for the analysis.

\section{RESULTS AND DISCUSSION}

\section{Molecular Descriptors of ETX}

The optimized structure of ETX at the B3LYP/6311G(d,p) level of theory, is shown in Figure 1, while all the descriptors obtained at the three different phases namely gas, water and ethanol are reported in Table 1. Observe that ETX exhibits the lower value of IP in the aqueous phase, which suggests that, in this phase, is easier remove an electron due to the solvation effect rendered by water due to its high polarity. The EA value for ETX is bigger in aqueous and ethanol medium in comparison to the gas phase, which suggests a minor electro affinity of ETX in this phase. Also, note that the values of $\eta$ and $S$ are similar in the aqueous and ethanol phases indicating that ETX is exhibiting a similar global stability, but in gas phase ETX result to be more stable. ETX has bigger values of $\omega$ at the aqueous and ethanolic phases, indicating a major propensity to accept electrons in comparison to those obtained at the gas phase. Similar results were obtained at the M06/6-311G(d,p) and $\omega \mathrm{B} 97 \mathrm{xd} / 6-311 \mathrm{G}(\mathrm{d}, \mathrm{p})$ levels of theory.

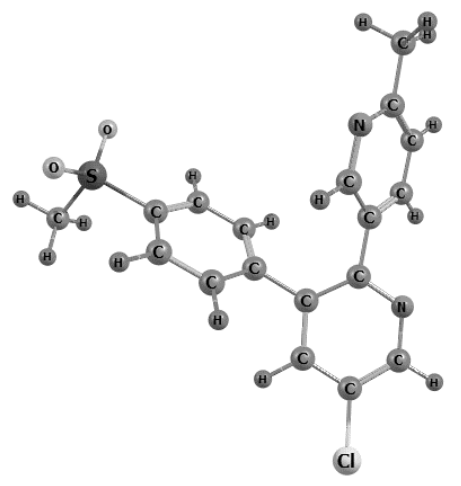

Figure 1. Optimized structure of ETX, at the B3LYP/6311G(d,p) level of theory

Table 1. Molecular descriptive parameters of ETX evaluated at the X/6$311 \mathrm{G}(\mathrm{d}, \mathrm{p}$ ) (where X=B3LYP, M06 and $\omega \mathrm{B} 97 \mathrm{XD}$ ) level of theory at the gas, water and ethanolic phases.

\begin{tabular}{|c|c|c|c|c|c|c|c|c|c|}
\hline & & B3LYP & & & M06 & & & $\omega \mathbf{\omega 9 7 X D}$ & \\
\hline & $\begin{array}{c}\mathbf{G a s} \\
(\mathbf{e V})\end{array}$ & $\begin{array}{c}\mathbf{H}_{2} \mathbf{O} \\
(\mathbf{e V})\end{array}$ & $\begin{array}{c}\mathbf{C}_{2} \mathbf{H}_{5} \mathbf{O H} \\
(\mathbf{e V})\end{array}$ & $\begin{array}{c}\mathbf{G a s} \\
(\mathbf{e V})\end{array}$ & $\begin{array}{c}\mathbf{H}_{2} \mathbf{O} \\
(\mathbf{e V})\end{array}$ & $\begin{array}{c}\mathbf{C}_{2} \mathbf{H}_{5} \mathbf{O H} \\
(\mathbf{e V})\end{array}$ & $\begin{array}{c}\mathbf{G a s} \\
(\mathbf{e V})\end{array}$ & $\begin{array}{c}\mathbf{H}_{2} \mathbf{O} \\
(\mathbf{e V})\end{array}$ & $\begin{array}{c}\mathbf{C}_{2} \mathbf{H}_{5} \mathbf{O H} \\
(\mathbf{e V})\end{array}$ \\
\hline $\mathrm{IP}$ & 8.12 & 6.56 & 6.60 & 8.18 & 6.63 & 6.67 & 8.45 & 6.89 & 6.93 \\
\hline $\mathrm{EA}$ & 0.72 & 2.08 & 2.04 & 0.74 & 2.12 & 2.08 & 0.39 & 1.82 & 1.78 \\
\hline$\eta$ & 3.70 & 2.24 & 2.28 & 3.72 & 2.26 & 2.30 & 4.03 & 2.53 & 2.58 \\
\hline $\mathrm{S}$ & 0.14 & 0.22 & 0.22 & 0.13 & 0.22 & 0.22 & 0.12 & 0.20 & 0.19 \\
\hline$\omega$ & 2.64 & 4.17 & 4.08 & 2.68 & 4.24 & 4.17 & 2.43 & 3.74 & 3.68 \\
\hline
\end{tabular}

\section{Molecular Docking}

For the molecular docking studies, a two-step validation process was performed. In the first one, RFX molecule was docked against COX-2 structure. The conformations found after the calculations were compared with the experimental crystallographic structure. The structure with the highest docking score $(-10.1 \mathrm{kcal} / \mathrm{mol})$ presents a perfect fit as shown in Figure 2a. In the second step of the validation process, ETX molecule was docked against COX-2 structure using the same parameters as for RFX. The conformations obtained were compared again with the experimental RFX structure. The conformation with the best binding score $(-7.4 \mathrm{kcal} / \mathrm{mol})$ presents the best fit against RFX where both molecules are almost overlaid (Figure $2 b$ ). This validation procedure suggests the conformation obtained for the interaction of ETX with the active site of COX-2 is correct.

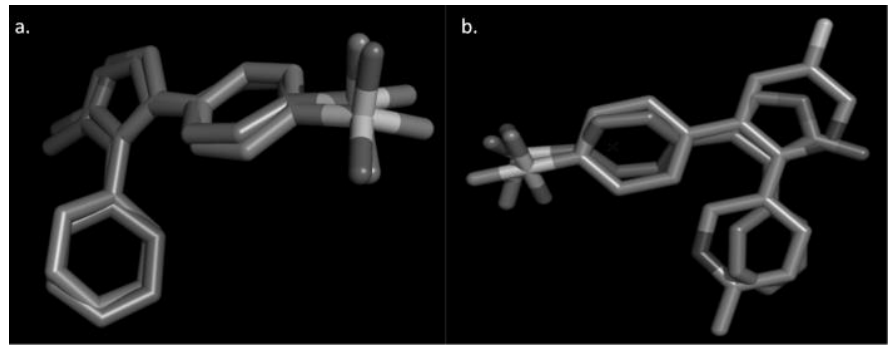

Figure 2. Comparison between RFX experimental structure (Blue) with a. RFX docked structure (Green) and b. ETX docked structure (Grey). Carbon atoms are present in the different colors depend on the molecule; oxygen is presented in red, sulphur in yellow and chlorine in light green.

With the ETX structure correctly fitted inside the active site of COX-2, a further analysis of the interaction was done. At first sight, the active site of COX2 seems to be very accessible for molecules such as ETX or RFX. Looking at the electrostatic potential surface of the enzyme, it is revealed that the active pocket is deep in the enzyme being more selective for this type of molecules (Figure 3).

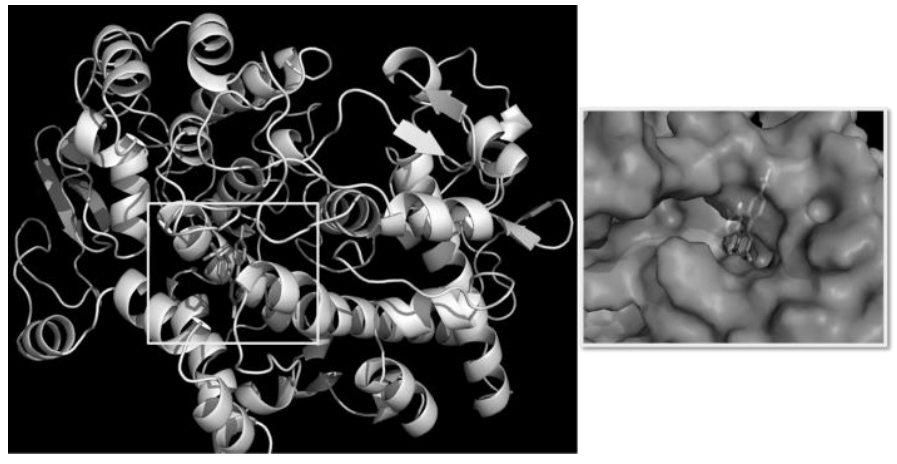

Figure 3. Human COX-2 structure bound to ETX.

The active site on COX-2 and COX-1 are very similar, probably, the main difference between them is the presence of a lateral chamber. This chamber located over the Arg120-Tyr355-Glu524 constriction made the active site 17\% bigger being able to fit bulkier molecules such as ETX, which COX-1 active site cannot. ${ }^{38,39}$ A joint analysis of the interaction was performed using Pymol and LigPlot+. With Pymol, all the hydrogen bonds between ETX and any residue of COX- 2 were estimated by measuring all the polar contact distances below $3.2 \AA$. The only hydrogen bond found for this interaction was between the hydrogen of the primary amine group of the sidechain of Arginine 513 and an oxygen atom in the sulphonyl group of ETX (Figure 4).
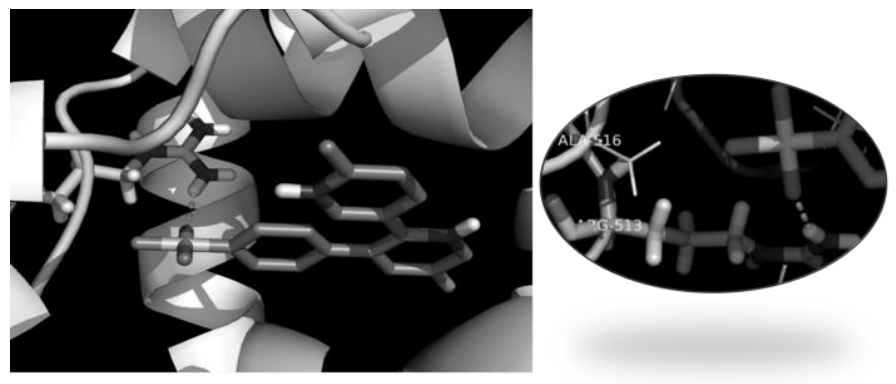

Figure 4. Hydrogen bond (yellow dotted line) between human COX-2 and ETX.

The analysis with LigPlot+ showed the same hydrogen bond between the same atoms with a distance of $2.98 \AA$. As the molecules are not fixed structures, the interaction found can happen between any of the amine groups of the sidechain of Arg513 with either Oxygen of the sulphonyl group of ETX. Furthermore, 
LigPlot+ allows spotting other interactions that may include hydrophobic interactions, $\pi$-stacking, weak hydrogen bonding, salt bridge, amide staking and cation- $\pi$ interactions. ${ }^{40-43}$ Note that ETX interacts with 19 residues in the active site of COX-2 (Figure 5a). Further a 3D analysis using Pymol can determine that most of the interactions are hydrophobic interactions, although nitrogen atoms in the pyridine cycles of ETX may form a salt bridge with oxygen atoms in Ala527 and Met522 respectively. Moreover, an amide stacking interaction may be happening between the amide bond of residues Gly527-Ala527 and the pyridine cycle (Figure 5b).
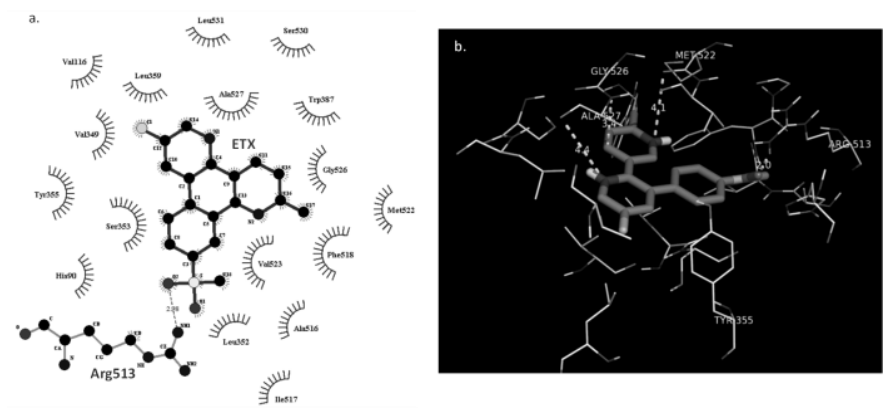

Figure 5. Interaction between ETX and COX-2 active site in 2D (a) and 3D (b).

\section{CONCLUSION}

The molecular structure study using molecular descriptors shows that Etoricoxib structure exhibits an enhanced structural activity in water and ethanol, which suggests this drug would be more active in biological solvents like in blood, tissues and places where COX-2 can be found in the human body. Pharmacologically, to inhibit COX-2, Etoricoxib interacts with 19 amino acid residues inside the COX-2 active site. From such interactions, only one hydrogen bond was found between the hydrogen of the primary amine group of the sidechain of Arg513 and an oxygen atom in the sulphonyl group of ETX. Most interactions found were hydrophobic interactions, one salt bridge interaction and an amide stacking interaction. The bigger size of the COX-2 active site in comparison with COX-1, allows Etoricoxib and coxib like molecules to selectively inhibit the COX-2 enzyme.

\section{ACKNOWLEDGMENTS}

LHMH gratefully acknowledges financial support from CONACYT (project CB(2015)-257823) and to the Universidad Autónoma del Estado de Hidalgo. Guanajuato National Laboratory (CONACyT 123732) is acknowledged for supercomputing resources. LHMH acknowledges to the SNI for the distinction of his membership and the stipend received.

\section{REFERENCES}

1. Y. Chai, L. Wang, Y. Bao, R. Teng, Y. Liu, C. Xie, Cryst. Growth Des. 19, 1660, ((2019)).

2. A. Okumu, M. DiMaso, R. Löbenberg, Eur. J. Pharm. Biopharm. 72(1), 91, ((2009)).

3. M. Palucki, Z. Lin, Y. Sun, Org. Process Res. Dev. (2005), 9(2) 141-148. DOI: 10.1021/op049802v

4. M. Rams-Baron, J. Pacult, A. Jedrzejowska, J. Knapik-Kowalczuk, M. Paluch, Mol. Pharm. (2018), 15(9), 3969-3978. DOI: 10.1021/acs.molpharmaceut. 8 b00428.

5. H. S. Omer Erkan Yapca, Mehmet Ibrahim Turan, Ismayil Yilmaz, Suleyman Salman, Mine Gulapoglu, J. Obstet. Gynaecol. Res. (2014), 40(6), 1674 1679. DOI: https://doi.org/10.1111/jog.12373.

6. M. Rams-Baron, Z. Wojnarowska, K. Grzybowska, M. Dulski, J. Knapik, K Jurkiewicz, W. Smolka, W. Sawicki, A. Ratuszna, M. Paluch, Mol. Pharm. (2015), 12(10), 3628-3638. DOI: 10.1021/acs.molpharmaceut.5b00351.

7. T. Zhang, L. Wang, Y. Bao, Q. Yang, L. Zhou, H. Hao, C. Xie, J. Pharm. Sci. (2018), 107(7), 1903-1910. DOI: 10.1016/j.xphs.(2018).03.007.

8. P. Grobelny, A. Mukherjee, G. R. Desiraju, CrystEngComm. (2012), 14, 5785-5794. DOI: 10.1039/c2ce06604a.

9. C. Rodríguez-Tinoco, M. Rams-Baron, K. L. Ngai, K. Jurkiewicz, J. Rodríguez-Viejo, M. Paluch, Phys. Chem. Chem. Phys. (2018), 20, 39393945. DOI: $10.1039 / \mathrm{c} 7 \mathrm{cp} 06445 \mathrm{~d}$
10. N. G. B. Agrawal, A. G. Porras, C. Z. Matthews, M. J. Rose, E. J. Woolf, B J. Musser, A. L. Dynder, K. E. Mazina, K. C. Lasseter, T. L. Hunt, et al., J. Clin. Pharmacol. (2003), 43(3), 268-276. DOI: 10.1177/0091270003251122. 11. S. Oniga, L. Pacureanu, C. Stoica, M. Palage, A. Crăciun, L. Rusu, E.-L. Crisan, C. Araniciu, Molecules. (2017), 22 (9), 1507. DOI: 10.3390/molecules22091507.

12. S. Sil, T. Ghosh, J. Neuroimmunol. (2018), 317, 15-23. DOI 10.1016/j.jneuroim.(2018).01.018.

13. T. Yapanoglu, F. Ozkaya, A. H. Yilmaz, R. Mammadov, F. K. Cimen, E. Hirik, Korean J. Physiol. Pharmacol. (2017), 21(5), 457-464. DOI https://doi.org/10.4196/kjpp.(2017).21.5.457.

14. A. Maheshwari, L. Badgujar, B. Phukan, S. L. Bodhankar, P. Thakurdesai, Eur. J. Pharmacol. (2011), 667(1-3), 230-7. DOI: 10.1016/j.ejphar.(2011).05.030.

15. R. Apak, M. Özyürek, K. Güçlü, E. Çapanołlu, J. Agr. Food Chem. (2016), 64(5), 1028-1045. DOI: 10.1021/acs.jafc.5b04743.

16. K. Anbazhakan, K. Sadasivam, R. Praveena, Struct. Chem. (2019), 30(4), 17 DOI: $10.1007 / \mathrm{s} 11224-018-1185-2$.

17. M. Leopoldini, T. Marino, N. Russo, M. Toscano, J. Phys. Chem. A. (2004), 108(2), 4916-4922. DOI: $10.1021 / \mathrm{jp} 037247 \mathrm{~d}$.

18. R. Praveena, K. Sadasivam, V. Deepha, R. Sivakumar, J. Mol. Struct. (2014), 1061(5), 114-123 DOI: 10.1016/j.molstruc.(2014).01.002.

19. K. Sadasivam, R. Kumaresan, Mol. Phys. (2011), 109(6), 839-852. https://doi.org/10.1080/00268976.2011.556576.

20. K. Sadasivam, R. Kumaresan, Spectrochim. Acta Part A Mol. Biomol. Spectrosc. (2011), 282-293. https://doi.org/10.1016/j.saa.2011.02.042

21. A. D. Becke, Phys. Rev. A (1988), 38(6), 3098-3100. DOI: https://doi.org/10.1103/PhysRevA.38.3098.

22. A. D. Becke, J. Chem. Phys. (1993), 98 (7), 5648-5652. https://doi.org/10.1063/1.464913.

23. Y. Zhao, D. G. Truhlar, Y. Zhao, D G. Truhlar, Theor Chem Acc. (2008) 120, 215-241. https://doi.org/10.1007/s00214-007-0310-x.

24. J. Da Chai, M. Head-Gordon, Phys. Chem. Chem. Phys. (2008), 10(44), 6615-6620. https://doi.org/10.1039/b810189b.

25. M. J. Frisch, G. W. Trucks, H. B. Schlegel, G. E. Scuseria, M. A. Robb, J. R. Cheeseman, G. Scalmani, V. Barone, B. Mennucci, G. A. Petersson, et al., (2009).

26. P. Geerlings, F. De Proft, W. Langenaeker, Chem. Rev. (2003), 103 (5), 1793-1874. DOI: $10.1021 / \mathrm{cr} 990029$ p.

27. R. G. Parr, W. Yang, Density-Functional Theory of Atoms and Molecules, First ed., Oxford University Press, New York (1989).

28. R. G. Parr, R. G. Pearson, J. Am. Chem. Soc. (1983), 105 (26), 7512-7516. DOI: $10.1021 / \mathrm{ja} 00364 \mathrm{a} 005$.

29. R. G. Pearson, J. Chem. Educ. (1987), 64 (7), 561. DOI: 10.1021/ed064p561.

30. R. G. Parr, P. K. Chattaraj, J. Am. Chem. Soc. (1991), 113 (5), 1854-1855. DOI: $10.1021 / \mathrm{ja} 00005 \mathrm{a} 072$

31. R. G. Pearson, J. Am. Chem. Soc. (1985), 107(24), 6801-6806

32. PyMOL, The PyMOL Molecular Graphics System, Version 2.0 Schrödinger, LLC.

33. E. F. Pettersen, T. D. Goddard, C. C. Huang, G. S. Couch, D. M. Greenblatt, E. C. Meng, T. E. Ferrin, J. Comput. Chem. (2004), 25(13), 1605.1612 DOI: $10.1002 /$ jcc. 20084 .

34. C. Steffen, K. Thomas, U. Huniar, A. Hellweg, O. Rubner, A. Schroer, $J$ Comput. Chem. (2010), 31(16), 2967-70. doi: 10.1002/jcc.21576.

35. D. S. Wishart, Y. D. Feunang, A. C. Guo, E. J. Lo, A. Marcu, J. R. Grant, T. Sajed, D. Johnson, C. Li, Z. Sayeeda, et al., Nucleic Acids Res. (2018), 46(D1), D1074-D1082 DOI: 10.1093/nar/gkx1037.

36. A. J. O. OLEG TROTT, A. Schroer, J. Comput. Chem. (2010), 31(2), 455461. DOI: $10.1002 /$ jcc.

37. R. A. Laskowski, M. B. Swindells, J. Chem. Inf. Model. (2011), 51(10), 2778 86. DOI: $10.1021 / \mathrm{ci} 200227 \mathrm{u}$.

38. D. L. DeWitt, Mol. Pharmacol. (1999), 55(4), 625-31

39. A. L. Blobaum, L. J. Marnett, J. Med. Chem. (2007), 50(7), 1425-1441. DOI $10.1021 / \mathrm{jm} 0613166$.

40. R. Ferreira De Freitas, M. Schapira, Medchemcomm. (2017), 8, 1970-1981 DOI: $10.1039 / \mathrm{c} 7 \mathrm{md} 00381 \mathrm{a}$

41. J. L. Medina-Franco, O. Méndez-Lucio, K. Martinez-Mayorga, Adv. Protein Chem. Struct. Biol. (2014), 96, 1-37. DOI: 10.1016/BS.APCSB.(2014).06.001.

42. J. E. Donald, D. W. Kulp, W. F. DeGrado, Proteins Struct. Funct. Bioinforma. (2011), 79(3), 898-915. DOI: 10.1002/prot.22927.

43. L. Yang, J. Zhang, X. Che, Y. Q. Gao, Methods Enzymol. (2016), 578, 169 212. DOI: 10.1016/BS.MIE.2016.05.031. 